\section{Intramolecular Reductive Coupling of Carbonyl-Tethered Oxime Ethers Promoted by Samarium Diiodide: A Powerful Method for the Stereoselective Synthesis of Aminocyclopentitols ${ }^{\dagger, \ddagger}$}

\author{
Jose Luis Chiara,* José Marco-Contelles, \\ Noureddine Khiar, Pilar Gallego, \\ Christine Destabel, and Manuel Bernabé
}

Instituto de Química Orgánica, C.S.I.C., Juan de la Cierva, 3, E-28006 Madrid, Spain

\section{Received August 2, 1995}

There is a growing interest in the synthesis of stereochemically pure aminocyclopentitols as key intermediates for the preparation of carbocyclic glycosidase inhibitors, ${ }^{1}$ carbocyclic nucleosides, ${ }^{2}$ and analogues. To this end, a series of carbocyclization strategies have been developed, a particularly interesting process being the intramolecular trapping of a radical by an oxime ether ${ }^{3}$ or by a hydrazone. ${ }^{4}$ The efficiency of these reactions lies in the extra stabilization of the intermediate aminyl radical by a lone pair in the adjacent oxygen or nitrogen atom.

We report here a new and efficient method for the preparation of polyfunctionalized aminocyclopentitols from readily available carbohydrate templates ${ }^{5}$ by a highly stereoselective intramolecular reductive coupling of carbonyl-tethered oxime ethers, promoted by samarium diiodide. ${ }^{6}$ This one-electron reducing agent has been used previously for the intermolecular reductive coupling of aldehydes and ketones with $O$-benzylformaldoxime ${ }^{7}$ and, more recently, for the corresponding intramolecular reductive coupling with $N, N$-diphenylhydrazones. ${ }^{4 \mathrm{~b}}$ In both cases, the addition of HMPA was found to be essential for the success of the coupling. Although the reported intermolecular cross-coupling with $O$-benzylformaldoxime failed completely with other aldoximes, ${ }^{7}$ we envisioned that the reaction could be feasible if performed intramolecularly.

The general strategy is outlined in Scheme 1. All the substrates for the cyclizations were easily prepared in two steps from readily available $O$-protected sugar hemiacetals by condensation with $O$-benzylhydroxylamine and

+ This work is dedicated to the memory of Prof. Félix Serratosa.

₹A part of this work was presented at the XVIIth International Carbohydrate Symposium, Ottawa, July 1994, Abstract B1.67.

(1) For some recent references, see: (a) Trost, B. M.; Van Vranken D. L. J. Am. Chem. Soc. 1993, 115, 444. (b) King, S. B.; Ganem, B. J. Am. Chem. Soc. 1994, 116, 562. (c) Knapp, S.; Purandare, A.; Rupitz K.; Withers, S. G. J. Am. Chem. Soc. 1994, 116, 7461 and references cited therein

(2) Marquez, V. E; Lim, M.-I. Med. Res. Rev. 1986, 6, 1.

(3) (a) Hart, D. J.; Seely, F. L. J. Am. Chem. Soc. 1988, 110, 1631 (b) Bartlett, P. A.; McLaren, K. L.; Ting, P. C. J. Am. Chem Soc. 1988 , 110, 1633. For recent examples, see: Booth, S. E.; Jenkins, P. R.; Swain, C. J.; Sweeney, J. B. J. Chem. Soc., Perkin Trans. 1 1994, 3499 and references cited therein.

(4) (a) Kim, S.; Kee, I. S. Tetrahedron Lett. 1993, 34, 4213. (b) Sturino, C. F.; Fallis, A. G. J. Am. Chem. Soc. 1994, 116, 7447. (c) Bowman, W. R.; Stephenson, P. T.; Terrett, N. K.; Young, A. R Tetrahedron Lett. 1994, 35, 6369.

(5) For a recent review on the use of carbohydrate templates for the preparation of carbocycles, see: Ferrier, R. J.; Middleton, S. Chem. Rev. 1993, 93, 2779 .

(6) For recent reviews on applications of samarium diiodide in organic synthesis, see: (a) Molander, G. A. Chem. Rev. 1992, 92, 29. (b) Imamoto, T. Lanthanides in Organic Synthesis; Academic Press: London, 1994

(7) Hanamoto, T.; Inanaga, J. Tetrahedron Lett. 1991, 32, 3555.

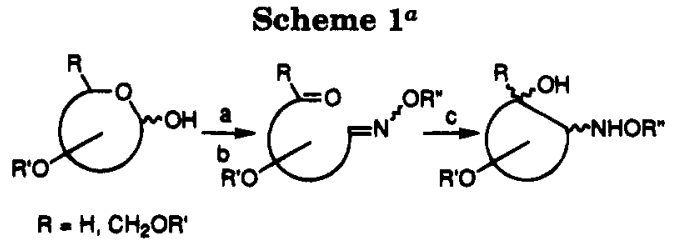

a Key: (a) $\mathrm{R}^{\prime \prime} \mathrm{ONH}_{2}$; (b) [O]; (c) $2 \mathrm{SmI}_{2}, 2 \mathrm{H}^{+}$.

Scheme $2^{a}$
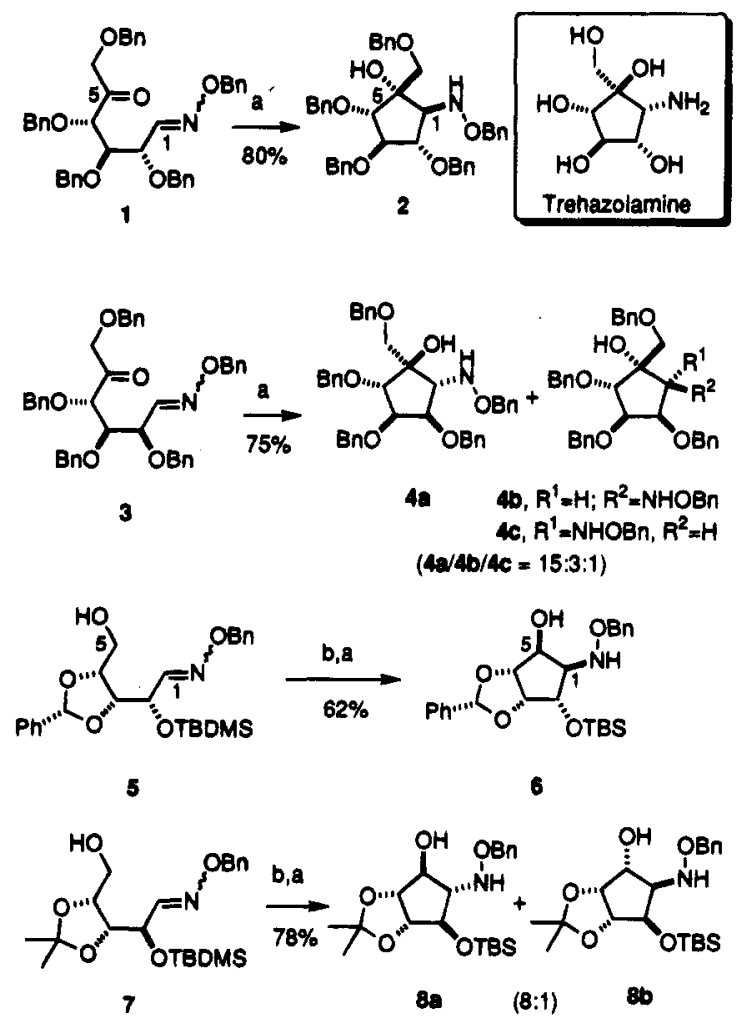

a Reagents and conditions: (a) $\mathrm{SmI}_{2}$ ( 3 equiv), $\mathrm{THF} / t-\mathrm{BuOH}$ $-25 \rightarrow 0{ }^{\circ} \mathrm{C}$; (b) (i) $(\mathrm{COCl})_{2}$, DMSO, THF, $-78^{\circ} \mathrm{C}$, (ii) $i$-Pr $\mathrm{Pr}_{2} \mathrm{NEt}$, $-78 \rightarrow 21^{\circ} \mathrm{C}$.

subsequent oxidation of the released hydroxyl group..$^{8,9}$ The ketone-oxime ether cross-coupling was studied first. Two substrates, 1 and 3, were prepared to this end, both differing in the configuration at $\mathrm{C}$-2, the center adjacent to the oxime ether group (Scheme 2).

When a $0.02 \mathrm{M}$ solution of 1 in THF was added to $\mathrm{SmI}_{2}$ in THF ( 3 equiv, $0.1 \mathrm{M}$ ) and $t$ - $\mathrm{BuOH}$ (2.5 equiv) at -25 ${ }^{\circ} \mathrm{C}$, the reductive coupling took place smoothly to afford the branched aminocyclopentitol 2 as a single diastereoisomer in good yield.9,10 Interestingly, this coupling proceeds in the absence of HMPA, in contrast to the analogous intermolecular case ${ }^{7}$ and to the corresponding coupling of hydrazones. ${ }^{4 b}$ It is also noteworthy that a single diastereoisomer is obtained from four possible stereochemical results. A similar approach has been reported very recently using a tributyltin hydride-induced reductive coupling of the corresponding $O$-methyloxime derivative of $1 .{ }^{11}$ In this case, a mixture of two diaste-

(8) See the supporting information for synthetic details.

(9) All new compounds showed correct microanalytical and spectroscopic data.

(10) The relative stereochemistry of the new centers was established by ${ }^{1} \mathrm{H}$ NMR and $2 \mathrm{D}$ NOESY studies.

(11) Kiguchi, T.; Tajiri, K.; Ninomiya, I.; Naito, T. Tetrahedron Lett. 1995, 36, 253 . 
reoisomeric aminocyclopentitols was obtained in $68 \%$ yield and 1.4:1 ratio. The minor isomer corresponds to that obtained by us using $\mathrm{SmI}_{2}$. Under the same conditions, ketone $\mathbf{3}$ afforded a mixture of three aminocyclopentitols, $4 \mathbf{a}-\mathbf{c},{ }^{9,10}$ in 15:3:1 ratio, ${ }^{12}$ respectively, and in good yield, from which the major diastereoisomer could be isolated in $60 \%$ yield by column chromatography. It should be noted that compounds 2 and $4 a-c$ can be readily converted, by hydrogenolysis, into epimers of trehazolamine (Scheme 2), the aminocyclopentitol aglycon of the trehalase inhibitor trehazoline. ${ }^{13}$ Thus, this sequence provides an efficient and very short entry into different enantiomerically pure analogues of this interesting aminocyclopentitol from readily available starting materials.

In order to explore the scope of the method we then examined the performance of aldehydes. For this purpose, alcohols 5 and 7 (Scheme 2) were prepared. As in the ketone case, both compounds have a similar substitution pattern but differ in the configuration at the carbon center adjacent to the oxime ether group. Initially, Swern oxidation of 5 and reductive cyclization of the isolated intermediate aldehyde gave cyclopentane $6^{9,10}$ as a single diastereoisomer in $32 \%$ overall yield from $\mathbf{5}$. However, the overall yield was greatly improved when the Swern oxidation and the $\mathrm{SmI}_{2}$ cyclization were performed in a one-pot sequence, thus avoiding the isolation of the intermediate aldehyde. ${ }^{14}$ Similarly, when compound $\mathbf{7}$ was subjected to this one-pot procedure, an inseparable mixture of two cyclopentanes $(\mathbf{8 a}, \mathbf{b})$ was obtained in 8:1 ratio, ${ }^{12}$ respectively, and good overall yield.

We have also observed that the stereochemistry of the cyclic products is independent of the geometry of the starting oxime ether. ${ }^{3 b}$ Although syn/anti mixtures of oximes have been used in all cases, a single stereochem. ical outcome was obtained in two examples (cyclizations of 1 and 5 ). ${ }^{15}$ In most cases, the major isomer shows a trans relationship between the hydroxyl and the $O$. benzylhydroxylamino groups and also between the latter and the alkoxy group at C-2.

The cyclic products could further react in situ with excess $\mathrm{SmI}_{2}$ at room temperature, after addition of water (20-25 equiv), ${ }^{16}$ by reduction of the $\mathrm{N}-\mathrm{O}$ bond ${ }^{17}$ to give the primary amine, ${ }^{18}$ thus further enhancing the utility of this methodology as demonstrated for 1 and 5 (Scheme

(12) Determined from the ${ }^{1} \mathrm{H}$ NMR of the crude reaction mixture

(13) For recent syntheses of this aminocyclitol or analogues, see ref 1d and the following: (a) Shiozaki, M.; Arai, M.; Kobayashi, Y.; Kasuya, A.; Miyamoto, S.; Furukawa, Y.; Takayama, T.; Haruyama, H. J. Org. Chem. 1994, 59, 4450. (b) Uchida, C.; Yamagishi, T.; Ogawa, S. J. Chem. Soc., Perkin Trans, 1 1994, 589 and references cited therein.

(14) We have recently described a similar one-pot sequence consisting of a Swern oxidation and a $\mathrm{SmI}_{2}$-induced pinacol coupling for the direct transformation of an alditol-derived 1,6-diol into an inositol derivative: Chiara, J. L.; Martín-Lomas, M. Tetrahedron Lett. 1994 35,2969 .

(15) In the case of 1 , the $E$ isomer could be isolated and submitted to cyclization separatedly, with the same stereochemical outcome as when a mixture enriched in the $Z$ isomer $(Z / E=76: 24)$ was used.

(16) For previous reports on $\mathrm{SmI}_{2}$-promoted reactions in the presence of water, see: Hanessian, S.; Girard, C. Synlett 1994, 861 and references cited therein.

(17) See ref $6 b, p 39$.

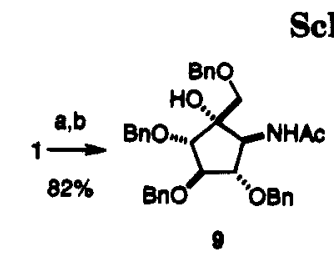

Scheme $3^{a}$

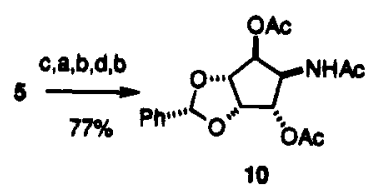

a Reagents and conditions: (a) (i) $\mathrm{SmI}_{2}$ (6 equiv), THF, -25 ${ }^{\circ} \mathrm{C}$, (ii) $\mathrm{H}_{2} \mathrm{O}$ (25 equiv), $-25 \rightarrow 22{ }^{\circ} \mathrm{C}$; (b) $\mathrm{Ac}_{2} \mathrm{O}$, py; (c) (COCl) DMSO, $\mathrm{Et}_{3} \mathrm{~N},-78 \rightarrow 22^{\circ} \mathrm{C}$; (d) TBAF, THF.

$3)$. This two-step one-pot sequence takes place in excellent yield in the case of 1 , and can be coupled with a preceding Swern oxidation step allowing the direct transformation of a 5-hydroxy oxime ether into a 2-aminocyclopentitol in a strikingly good overall yield, as exemplified for $5 .^{19}$

In conclusion, we have shown that the intramolecular reductive coupling of carbonyl-tethered oxime ethers can be promoted by samarium diiodide under very mild conditions, in the absence of HMPA, in good chemical yield and stereoselectivity. Moreover, we have shown that the reductive coupling reaction can be performed in a one-pot sequence with a prior Swern oxidation step, allowing the direct transformation of hydroxyl-tethered oxime ethers into the corresponding aminocyclitols, a process which is especially advantageous when the cyclization involves an aldehyde. The resultant cyclic hydroxylamine ethers can be efficiently converted in situ to the corresponding aminocyclitols by $\mathrm{N}-\mathrm{O}$ reductive cleavage promoted by excess samarium diodide and water. These processes have been applied to highly functionalized substrates derived from carbohydrates, providing a short and selective entry to carbocyclic amino polyols of varying regio- and stereochemistry. The extension of these studies to the synthesis of carbocyclic glycosidase inhibitors, aminoinositols, and analogues is in progress.

Acknowledgment. Financial support by DGICYT (Grant Nos. PB93-0127-C02-01 and SAF94-0818-C0202), CICYT (Grant No. CE93-0023), Comunidad Autónoma de Madrid (Grant No. AE-0094/94), and the European Union (Human Capital and Mobility Programme; contract ERBCHRXCT 92-0027) is gratefully acknowledged.

Supporting Information Available: Experimental procedures and characterization data for all new compounds and tables of ${ }^{1} \mathrm{H}$ NMR and 2D NOESY cross-peak intensities for cyclopentane products ( 8 pages).

\section{JO951391G}

(18) To facilitate isolation, the crude reaction mixtures were treated with $\mathrm{Ac}_{2} \mathrm{O}$ and pyridine at room temperature, affording the $N, O$. diacetylated compounds. The tertiary hydroxyl of compound $\mathbf{9}$ is unreactive under these conditions, and when the acetylation was performed in the presence of catalytic DMAP a complex mixture of products resulted.

(19) In the case of 5 , the sequence of Swern oxidation, reductive carbocyclization and $\mathrm{N}-\mathrm{O}$ reductive cleavage produced an inseparable mixture of two products ( $4: 1$ ratio) in $86 \%$ overall yield, which resulted from partial silyl migration to nitrogen during the reductive cleavage step. Desilylation of the mixture with TBAF in THF and acetylation afforded 10 as a single product in $92 \%$ yield. 